\section{POS1124 IDENTIFYING POTENTIAL CLASSIFICATION CRITERIA FOR CALCIUM PYROPHOSPHATE DEPOSITION DISEASE (CPPD): RESULTS FROM THE INITIAL PHASES}

S. Tedeschi ${ }^{1}$, T. Pascart ${ }^{2}$, A. Latourte ${ }^{3}$, C. Godsave ${ }^{4}$, B. Kundaki ${ }^{4}$, R. Naden ${ }^{5}$ W. Taylor ${ }^{6}$, N. Dalbeth ${ }^{7}$, T. Neogi ${ }^{8}$, F. Perez-Ruiz ${ }^{9}$, A. Rosenthal ${ }^{10}$, F. Becce ${ }^{11}$, E. Pascual ${ }^{12}$, M. Andrés ${ }^{12}$, T. Bardin ${ }^{3}$, M. Doherty ${ }^{4}$, H. K. Ea ${ }^{3}$, G. Filippou ${ }^{13}$, J. Fitzgerald ${ }^{14}$, M. Gutierrez ${ }^{15}$, A. lagnocco $0^{16}$, T. Jansen $^{17}$, M. Kohler ${ }^{18}$, F. Lioté ${ }^{3}$, M. Matza ${ }^{18}$, G. Mccarthy ${ }^{19}$, R. Ramonda ${ }^{20}$, A. Reginato ${ }^{21}$, P. Richette ${ }^{3}$, J. Singh ${ }^{22}$, F. Sivera ${ }^{23}$, A. So ${ }^{24}$, L. Stamp ${ }^{25}$, J. Yinh ${ }^{18}$, C. Yokose ${ }^{18}$ R. Terkeltaub ${ }^{26}, \mathrm{H}$. Choi ${ }^{18}$, A. Abhishek ${ }^{4} .{ }^{1}$ Brigham and Women's Hospital, Rheumatology, Boston, United States of America; ${ }^{2}$ Lille Catholic University, Rheumatology, Lille, France; ${ }^{3}$ Hôpital Lariboisière, Rheumatology, Paris, France; ${ }^{4}$ University of Nottingham, Academic Rheumatology, Nottingham, United Kingdom, ${ }^{5}$ Auckland City Hospital, Medicine, Auckland, New Zealand; ${ }^{6}$ University of Otago, Medicine, Wellington, New Zealand; ${ }^{7}$ University of Auckland, Medicine, Auckland, New Zealand; ${ }^{8}$ Boston University School of Medicine, Rheumatology, Boston, United States of America; ${ }^{9}$ Cruces University Hospital, Rheumatology, Basque Country, Spain; ${ }^{10}$ Medical College of Wisconsin, Rheumatology, Milwaukee, United States of America; ${ }^{11}$ Lausanne University Hospital, Radiology, Lausanne, Switzerland; ${ }^{12}$ Hospital General Universitario de Alicante, Rheumatology, Alicante, Spain; ${ }^{13}$ Luigi Sacco University Hospital, Rheumatology, Milan, Italy; ${ }^{14}$ UCLA David Geffen School of Medicine, Rheumatology, Los Angeles, United States of America; ${ }^{15}$ Instituto Nacional de Rehabilitacion, Musculoskeletal and Rheumatic Disorders, Mexico City, Mexico; ${ }^{16}$ Università degli Studi di Torino, Academic Rheumatology, Turin, Italy; ${ }^{17}$ VieCuri Medical Center, Rheumatology, Venlo, Netherlands; ${ }^{18}$ Massachusetts General Hospital, Rheumatology, Boston, United States of America: ${ }^{19}$ Mater Misericordiae University Hospital, Rheumatology, Dublin, Ireland; ${ }^{20}$ University of Padova, Rheumatology, Padova, Italy; ${ }^{21}$ Brown University School of Medicine, Rheumatology, Providence, United States of America; ${ }^{22}$ University of Alabama at Birmingham, Rheumatology, Birmingham, United States of America; ${ }^{23}$ Universidad Miguel Hernandez, Rheumatology, Elche, Spain; ${ }^{24}$ University Hospital of Lausanne, Musculoskeletal Medicine, Lausanne, Switzerland; ${ }^{25}$ University of Otago, Medicine, Christchurch, New Zealand; ${ }^{26}$ San Diego VA Healthcare Service and UCSD, Rheumatology, San Diego, United States of America

Background: Classification criteria for calcium pyrophosphate deposition disease (CPPD) will facilitate clinical research on this common crystalline arthritis. ACR/EULAR are jointly sponsoring development of CPPD classification criteria using a multi-phase process.

Objectives: To report preliminary results from the first two phases of a fourphase process for developing CPPD classification criteria.

Methods: CPPD classification criteria development is overseen by a 12-member Steering Committee. Item generation (Phase I) included a scoping literature review of five literature databases and contributions from a 35-member Combined Expert Committee and two Patient Research Partners. Item reduction and refinement (Phase II) involved a Combined Expert Committee meeting, discussions among Clinical, Imaging, and Laboratory Advisory Groups, and an item rating exercise to assess the influence of individual items toward classification. The Steering Committee reviewed the modal rating score for each item (range -3 [strongly pushes away from CPPD] to +3 [strongly pushes toward CPPD]) to determine items to retain for future phases of criteria development.

Results: Item generation yielded 420 items (312 from the literature, 108 from experts/patients). The Advisory Groups eliminated items they agreed were unlikely to distinguish between CPPD and other forms of arthritis, yielding 127 items for the item rating exercise. Fifty-six items, most of which had a modal rating of $+/-2$ or 3 , were retained for future phases (see Table 1 ). As numerous imaging items were rated +3 , the Steering Committee recommended focusing on imaging of the knee, wrist, and one additional affected joint for calcification suggestive of CPP crystal deposition.

Conclusion: The ACR/EULAR CPPD classification criteria working group has adopted both data- and expert-driven approaches, leading to 56 candidate items broadly categorized as clinical, imaging, and laboratory features. Remaining steps for criteria development include domain establishment, item weighting through a multi-criteria decision analysis exercise, threshold score determination, and criteria validation.
Table 1. Categories of items retained for future phases of classification criteria development

Age in decade at symptom onset

Acute inflammatory arthritis (e.g. knee, wrist, $1^{\text {st }}$ MTP joint $\left.{ }^{\star}\right)$

Recurrence and pattern of joint involvement (e.g. 1 self-limited episode, $>1$ self-limited episode)

Physical findings (e.g. palpable subcutaneous tophus* ${ }^{\star}$, psoriasis*)

Co-morbidities and family history (e.g. Gitelman disease, hemochromatosis, familial CPPD) Osteoarthritis location and features (e.g. $2^{\text {nd }}$ or $3^{\text {rd }}$ MCP joint, wrist)

Synovial fluid findings (e.g. CPP crystals present, CPP crystals absent on 1 occasion* or 2 occasions ${ }^{\star}$, monosodium urate crystals present ${ }^{\star}$ )

Laboratory findings (e.g. hypomagnesemia, hyperparathyroidism, rheumatoid factor*, anti-CCP*)

Plain radiograph: calcification in regions of fibro- or hyaline cartilage ${ }^{+}$

Plain radiograph: calcification of the synovial membrane/capsule/tendon

Conventional CT: calcification in regions of fibro- or hyaline cartilage ${ }^{+}$

Conventional CT: calcification of the synovial membrane/capsule/tendon ${ }^{+}$

Ultrasound: CPP crystal deposition in fibro- or hyaline cartilage ${ }^{+}$

Ultrasound: CPP crystal deposition in synovial membrane/capsule/tendons ${ }^{+}$

Dual-energy CT: CPP crystal deposition in fibro- or hyaline cartilage ${ }^{+}$

Dual-energy CT: CPP crystal deposition in synovial membrane/capsule/tendon

${ }^{*}$ Potential negative predictor ${ }^{+}$Assessed in the knee, wrist, and/or 1 additional affected joint

Disclosure of Interests: Sara Tedeschi Consultant of: NGM Biopharmaceuticals, Tristan Pascart: None declared, Augustin Latourte Consultant of: Novartis, Cattleya Godsave: None declared, Burak Kundaki: None declared, Raymond Naden: None declared, William Taylor: None declared, Nicola Dalbeth Speakers bureau: Abbvie and Janssen, Consultant of: AstraZeneca, Dyve, Selecta, Horizon, Arthrosi, and Cello Health, Tuhina Neogi: None declared, Fernando PerezRuiz: None declared, Ann Rosenthal: None declared, Fabio Becce Consultant of: Horizon Therapeutics, Grant/research support from: Siemens Healthineers, Eliseo Pascual: None declared, Mariano Andrés: None declared, Thomas Bardin: None declared, Michael Doherty: None declared, Hang Korng Ea: None declared, Georgios Filippou: None declared, John FitzGerald: None declared, Marwin Gutierrez: None declared, Annamaria lagnocco: None declared, Tim Jansen Speakers bureau: Abbvie, Amgen, BMS, Grunenthal, Olatec, Sanofi Genzyme, Consultant of: Abbvie, Amgen, BMS, Grunenthal, Olatec, Sanofi Genzyme, Minna Kohler Speakers bureau: Lilly, Consultant of: Novartis, Frederic Lioté: None declared, Mark Matza: None declared, Geraldine McCarthy Consultant of: PK Med, Roberta Ramonda: None declared, Anthony Reginato: None declared, Pascal Richette: None declared, Jasvinder Singh Speakers bureau: Simply Speaking, Consultant of: Crealta/Horizon, Medisys, Fidia, UBM LLC, Trio health, Medscape, WebMD, Adept Field Solutions, Clinical Care options, Clearview healthcare partners, Putnam associates, Focus forward, Navigant consulting, Spherix, Practice Point communications, Francisca Sivera: None declared, Alexander So: None declared, Lisa Stamp: None declared, Janeth Yinh: None declared, Chio Yokose: None declared, Robert Terkeltaub Consultant of: Sobi, Horizon Therapeutics, Astra-Zeneca, Selecta, Grant/research support from Astra-Zeneca, Hyon Choi: None declared, Abhishek Abhishek Consultant of: NGM Biopharmaceuticals.

DOI: 10.1136/annrheumdis-2021-eular.469

\section{POS1125 DUAL ENERGY CT HAS PROGNOSTIC VALUE IN GOUT BEYOND STANDARD CLINICAL MEASURES: A BEST EVIDENCE SYNTHESIS}

S. Stauder ${ }^{1}$, P. M. Peloso ${ }^{2} .{ }^{1}$ Florida State University, Medical, Tallahassee, United States of America; ${ }^{2}$ Horizon Therapeutics plc, Research and Development, Deerfield, United States of America

Background: Dual Energy CT Scan (DECT) can detect monosodium urate crystal deposits in multiple tissues. EULAR gout guidelines (Richette, 2020) recognized the value of DECT in making a clinical diagnosis when joint aspiration is difficult. DECT shows crystal deposits in almost $50 \%$ of gout patients without tophi (Dalbeth, 2017). Tophi are known to predict all-cause and cardiovascular mortality (Vincent 2017, Perez-Ruiz 2013) and it is plausible that DECT could as well. A prognostic measure should be reliable and valid. DECT validity would be evident for death, disability and distress.

Objectives: This study used a best evidence synthesis approach to synthesize the evidence for DECT as a prognostic measure in gout.

Methods: PUBMED and EMBASE were searched from initiation to December 2019; keywords (Dual Energy Computed Tomography OR DECT, gout, 
tophaceous gout, chronic gout, monosodium urate crystals OR monosodium urate burden OR tophi OR monosodium urate volume OR flares OR pain OR distress OR death OR disability OR function). Human studies in English were considered. Titles, abstracts and full articles were reviewed. A manual search of secondary sources was conducted. Key gaps identified were considered throughout 2020 when reviewing emerging articles and presentations. Data extraction was conducted by both authors; data presented represents consensus.

Results: Of 344 articles, 81 titles/abstracts met screening inclusion criteria (24\%) in the 2019 search; review of the full manuscript led to 41 articles selected (51\%). Additionally, 3 key papers and 2 ACR 2020 presentations were identified through 2020. DECT is highly reliable with inter-class correlation coefficients $>0.9$. DECT has content validity. Dalbeth (2015) showed DECT and X-Rays findings correlated in tophaceous patients, $r=0.70, p<0.001$. Hand function correlates with DECT burden, with $r^{2}=0.59, p=0.024$ (Dalbeth 2007). Dalbeth (2017) showed DECT associated with greater flares at 3 and 12 months $(p<0.01)$ in 152 patients. Pascart (2018) confirmed that subjects with flares had nearly doubled DECT feet volumes $\left(0.9\right.$ vs $\left.2.1 \mathrm{~cm}^{3}, \mathrm{p}=0.05\right)$ versus those not flaring. Dalbeth (2017) showed abnormal DECT scans occurred in $47 \%$ of patients with normal uric acid $(<6.0 \mathrm{mg} / \mathrm{dL})$ without palpable tophi and in $90 \%$ with elevated uric acid and palpable tophi. DECT is very sensitive to change (Araujo 2015) with $95 \%$ volume reduction in 152 patients on pegloticase treated up to 12 months. Three studies show DECT is correlated to cardiovascular risk factor prevalence (Pascart 2020, Gamala 2018, Lee 2017). Marty-Ané reported that DECT volume predicts mortality (Marty-Ané ACR 2020). Limited evidence from 3 studies suggests that the minimum important volume for DECT is $1.0 \mathrm{~cm}^{3}$ at feet and ankles, including Pascart 2020.

Conclusion: DECT imaging is highly reliable, has evidence for content validity and is highly sensitive to change. DECT appears to predict future gout flares, cardiovascular risk factor prevalence and mortality. Minimum important DECT volume approximates $1.0 \mathrm{~cm}^{3}$. DECT requires further study but appears to be a relevant outcome for clinical trials and staging gout patients.

\section{REFERENCES:}

\begin{tabular}{lcc}
\hline Authors & Journal, Volume, Issue & Year \\
\hline Araujo, E. G., Bayat, S., et al. & RMD Open & 2015 \\
Dalbeth, N., Nicolaou, S., et al. & Ann Rheum Dis, 77(3) & 2017 \\
Dalbeth, N., Aati, O., et al. & Ann Rheum Dis, 74(6) & 2015 \\
Dalbeth, N., Collis, J., et al. & Rheumatology, 46(12) & 2007 \\
Gamala, M., Linn-Rasker, S. P., et al. & Clinical Rheumatol, 37(7) & 2018 \\
Lee, K., Ryu, S., et al. & Clinical Rheumatol, 37 & 2017 \\
Marty-Ané, A., Norberciak, L., et al. & Arthritis Rheumatol 72 (supp 10) & 2020 \\
& [abstract \#0954] ACR 2020. & \\
Pascart, T., Ramon, A., et al. & J Clin Med, 9(5) & 2020 \\
Pascart, T., Capon, B., et al. & Arthritis Res and Therapy, 20(1) & 2018 \\
Perez-Ruiz, F., Martínez-Indart, L., et al. & Ann Rheum Dis, 73(1) & 2013 \\
Richette, P., Doherty, M., et al. & Ann Rheum Dis, 79(1) & 2020 \\
Vincent, Z., Gamble, G., et al. & J Rheumatol, 44 (3) & 2017 \\
\hline
\end{tabular}

Disclosure of Interests: Sally Stauder: None declared, Paul M. Peloso Shareholder of: Horizon Therapeutics plc, Employee of: Horizon Therapeutics plc. DOI: 10.1136/annrheumdis-2021-eular.872

\section{POS1126 PREVALENCE OF HYPOPHOSPHATASIA IN ADULT RHEUMATOLOGY PATIENTS SCREENED BY LOW LEVELS OF ALKALINE PHOSPHATASE}

P. Karakostas ${ }^{1}$, R. Dolscheid-Pommerich ${ }^{2}$, M. Hass ${ }^{3}$, P. Brossart ${ }^{4}$, V. Schäfer ${ }^{4}$. ${ }^{1}$ University Hospital of Bonn, Germany, Clinic of Internal Medicine III, Oncology, Hematology, Rheumatology and Clinical Immunology, Bonn, Germany;

${ }^{2}$ University Hospital of Bonn, Germany, Institute of Clinical Chemistry and Clinical Pharmacology, Bonn, Germany; ${ }^{3}$ Center for Blood Coagulation Disorders and Transfusion Medicine Bonn, Germany, Center for Blood Coagulation Disorders and Transfusion Medicine Bonn, Germany, Bonn, Germany; ${ }^{4}$ University Hospital of Bonn, Germany, Clinic of Internal Medicine III, Oncology, Hematology, Rheumatology and Clinical Immunology, Bonn, Germany

Background: Hypophosphatasia (HPP) is a genetic disease caused by one or more mutations in the alkaline phosphatase (ALP) gene, which encodes tissue-specific ALP and affects the mineralization process. Accordingly, arthralgia, fractures, and dental abnormalities have been reported in adults, and fatal courses in children. This metabolic disorder is commonly misdiagnosed with other more prevalent bone diseases due to its low prevalence and lack of recognition (i.e. chrondrocalcinosis). However, no epidemiological studies on the prevalence of HPP in the rheumatological patient population have been available to date.

Objectives: To identify the prevalence of HPP in rheumatological patients screened for persistent low levels of ALP and association with mutations in the ALP gene.
Methods: All adult rheumatology patients were screened for pathological low levels of ALP (<35 IU/L) between January 1, 2017 and June 30, 2019 at the Department of Rheumatology, Clinic of Internal Medicine III, University Hospital Bonn, Germany. Medical files of patients with pathological low ALP levels were then reviewed for clinical signs and symptoms as well as results of genetic testing for HPP (full sequencing using Next Generation Sequencing).

Results: In total, 2,289 rheumatology patients were screened for low ALP levels. In 60 patients (2.62 \%), pathological low ALP levels were identified, while in 30 of these $(1.31 \%)$, persistent low ALP levels were detected. In 19 of the 30 patients, genetic tests for ALPL gene mutations were done. Seven out of 19 patients $(36.84 \%$ ) had HPP-related symptoms (fracture, dental abnormalities) with normal bone densitometry, while four of these patients $(21.05 \%)$ had a history of fracture and three patients (15.78\%) showed dental abnormalities. In addition to the typical HPP signs and symptoms, 13 patients $(68.42 \%)$ showed mutations in the ALPL gene. One of the ALP mutations was found to be a nove genetic variant, classified as pathological. Interestingly, no association with chondrocalcinosis was detected.

Conclusion: In summary, it can be concluded that HPP is an under-diagnosed condition with a higher proportion of affected rheumatologic patients than previously thought (at least 0.56 percent of rheumatologic patients vs. 0,01 percent in a spanish healthy population). If we replicate these numbers for the german population (83 million, 5 percent of whom suffer from rheumatic conditions) the yield is approximately 4.15 million. This possibly indicates that 23,240 potential cases of HPP are currently not diagnosed. Therefore, implementation of a protocol in clinical practice to prevent underdiagnosis of HPP and to treat this disease appropriately is essential.

\section{REFERENCES:}

[1] García-Fontana C, Villa-Suárez JM, Andújar-Vera F et al (2019) Epidemiological, Clinical and Genetic Study of Hypophosphatasia in A Spanish Population: Identification of Two Novel Mutations in The Alpl Gene. Sci Rep. https://doi.org/10.1038/s41598-019-46004-2

[2] Mornet E, Yvard A, Taillandier A et al (2011) A molecular-based estimation of the prevalence of hypophosphatasia in the European population. Ann Hum Genet 75:439-445. https://doi.org/10.1111/j.1469-1809.2011.00642.x

\section{$\mathrm{N}=2289$ adults with \\ rheumatologic diseases}
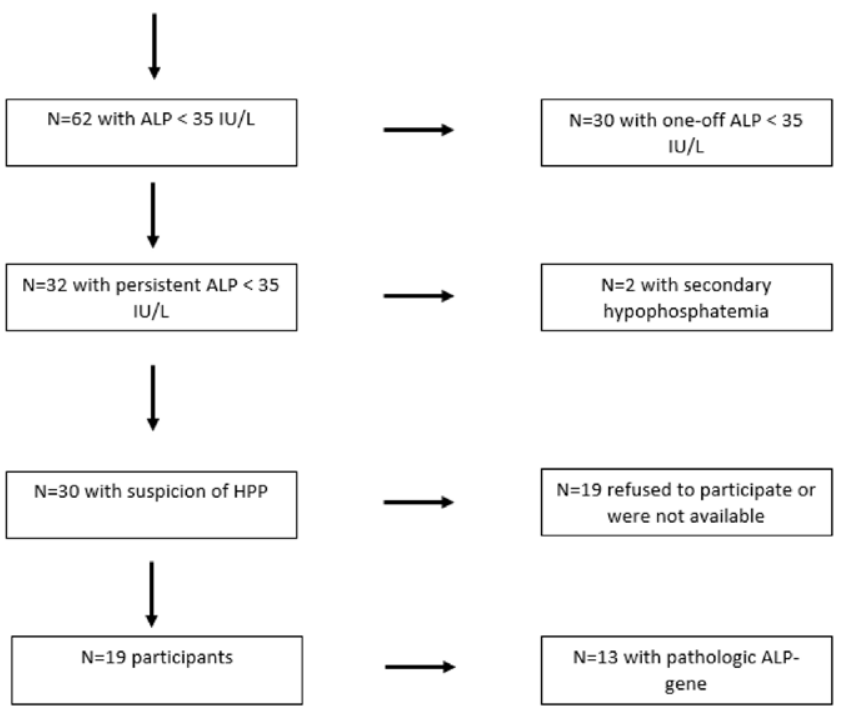

Figure 1.

Flow chart of identification of potential hypophosphatasia (HPP) patients from an adult rheumatology population. Threshold alkaline phosphatase $<35 \mathrm{IU} / \mathrm{L}$ at $37^{\circ} \mathrm{C}$. ALP: alkaline phosphatase

Disclosure of Interests: Pantelis Karakostas: None declared, Ramona Dolscheid-Pommerich: None declared, Moritz Hass: None declared, Peter Brossart: None declared, Valentin Schäfer Speakers bureau: AbbVie, Novartis, BMS Chugai, Celgene, Medac, Sanofi, Lilly, Hexal, Pfizer, Janssen, Roche, Schire Onkowissen, Royal College London, Consultant of: Novartis, Chugai, AbbVie, Celgene, Sanofi, Lilly, Hexal, Pfizer, Amgen, BMS, Roche, Gilead, Medac, Grant research support from: Novartis, Hexal, Lilly, Roche, Celgene, Universität Bonn. DOI: 10.1136/annrheumdis-2021-eular.1082 